\title{
INDUCTION AND GROWTH OF FLAX GENOTROPHS
}

\author{
ALAN DURRANT \\ Department of Agricultural Botany, University College of Wales, Aberystwyth
}

Received 22.x.70

\section{InTRODUCTION}

Heritable changes are induced in flax plants when grown in different environments. Most of the changes have been induced in the flax variety Stormont Cirrus grown in different fertiliser combinations in the greenhouse and in the field (Durrant, 1962a). When the progeny of the treated plants

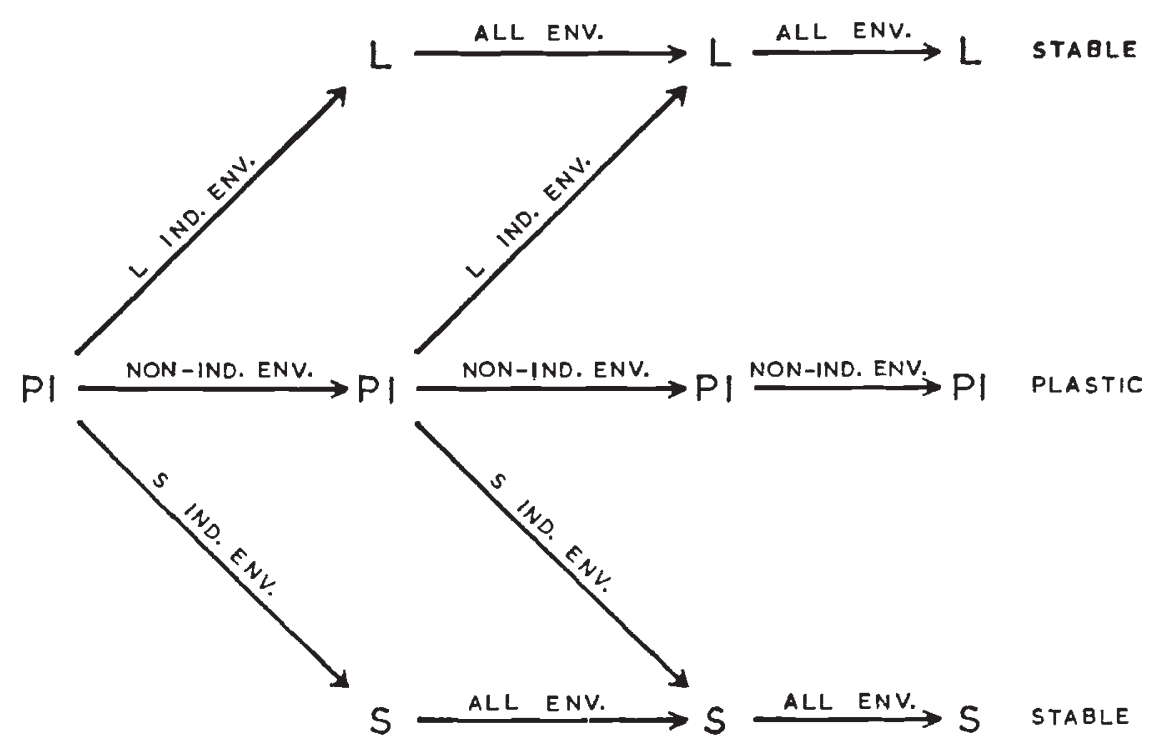

Fig. 1.-Environmental induction of $\mathrm{L}$ and $\mathrm{S}$ stable genotrophs from the $\mathrm{Pl}$ genotroph.

L ind. env: L-inducing environment

$S$ ind. env: S-inducing environment

non-ind. env: non-inducing environment

are compared in the next generation under uniform conditions the plants vary in size depending on the fertiliser combinations applied to their parents. They range from a large type about twice the size of the original variety to a small type about half the size of the original variety. When the range of induced types are themselves grown in different environments the largest and smallest induced types breed true but those of intermediate size repeat the process giving true breeding large and small types, and intermediate types in which further changes can be induced, as shown in fig. 1. Consequently there are three distinct types of plants; an intermediate plastic genotroph $(\mathrm{Pl})$ in which heritable changes can be induced; a large stable genotroph (L) induced from $\mathrm{Pl}$ which breeds true irrespective of the subsequent environment; and a small stable genotroph (S) which also breeds true irrespective of the subsequent environment. Expressed in another way, the un- 
differentiated $\mathrm{Pl}$ state undergoes permanent change to either the $\mathrm{L}$ or $\mathrm{S}$ differentiated state.

The genetic difference between $\mathrm{L}$ and $\mathrm{S}$ is primarily nuclear since there is no transmission through reciprocal grafts and reciprocal crosses give equilinear inheritance in the $F_{1}$. They have nevertheless arisen from genuine induced genetic changes rather than from some unique system of selection of true breeding homozygous types from heterozygous material, for the following reasons. (1) The induced changes have been repeated in separate experiments. (2) The plants within each genotroph are uniform. (3) The $F_{1}$ of crosses between $\mathrm{L}$ and $\mathrm{S}$ are genetically unstable giving increased variation in the $\mathrm{F}_{1}$ and significant positive correlation between $\mathrm{F}_{1}$ plants and their $\mathrm{F}_{2}$ family means (Durrant, 1962b). (4) L has 16 per cent. more nuclear DNA than S as judged by feulgen photometry; Pl has an intermediate amount. Plant weight is therefore correlated with the amount of nuclear DNA. The change in amount of nuclear DNA can be measured week by week during induction over the first five weeks of growth (Evans, Durrant and Rees, 1966; Evans, 1968). It is impossible to ascribe this to the selection of different genetic types. (5) Associated changes can be induced in another character, presence or absence of hairs on the septa in the capsules (Durrant and Nicholas, 1970). This is determined by a major gene which is also unstable in crosses between the genotrophs.

The large difference between the $\mathrm{L}$ and $\mathrm{S}$ genotrophs induced from $\mathrm{Pl}$ is uniform among the plants and it would seem that any set of environmental conditions approximating to that which was successful the first time would be effective in inducing the changes. Even if the fully induced changes were not realised at least the changes should be sufficiently large for practical purposes. This is not so. The inducing environments need to be carefully defined. It follows that care is needed as well in selecting the best environment for multiplying up seed stocks of the $\mathrm{Pl}$ genotroph otherwise unwanted induced changes will occur. Figure 2 shows the results of one sequence of induction experiments over the years. The large/small ratio, which is the mean weight of the larger plants resulting from induction in one environment divided by the mean weight of the smaller plants from induction in another, is plotted against the year in which the inducing environments were applied. The parental generation, i.e. the plants receiving the inducing environment is symbolised $C_{0}$, and the subsequent generations $C_{1}, C_{2}$, etc. Normally the $C_{2}$ generation is used for assessing induced change to diminish maternal effects, but where no obvious change occurred in $C_{1}$, no $C_{2}$ was grown and $C_{1}$ is entered instead in fig. 2, i.e. when the ratio is approximately equal to one. The large induced type should be three or four times the size of the small induced type provided they are compared under appropriate conditions (section 4). The experiments were done on a large scale and the results in fig. 2 are statistically valid. They show great variation in the effectiveness of the inducing treatments over years and an ominous downward trend throughout. This trend must be due to the inducing environments becoming increasingly ineffective, or to a change and gradual loss in plasticity of plants of the $\mathrm{Pl}$ genotroph during seed multiplication over years, or to both causes. The problem is not easily resolved over a short period of time because changes in most environmental factors likely to be responsible (e.g. decline in soil fertility) are correlated with the number of generations of seed multiplication, and because of the undesirability of comparing seed of 
different ages, the necessity for testing the progeny of the treated plants under optimal environmental conditions (section 4), changes in technique with the dispersal of limited greenhouse facilities for other studies and the time required (normally three years) for each experiment. This paper describes a number of other, earlier experiments, on the genotrophs, environments and their interactions, which serve as a background to more recent studies which are to be published.

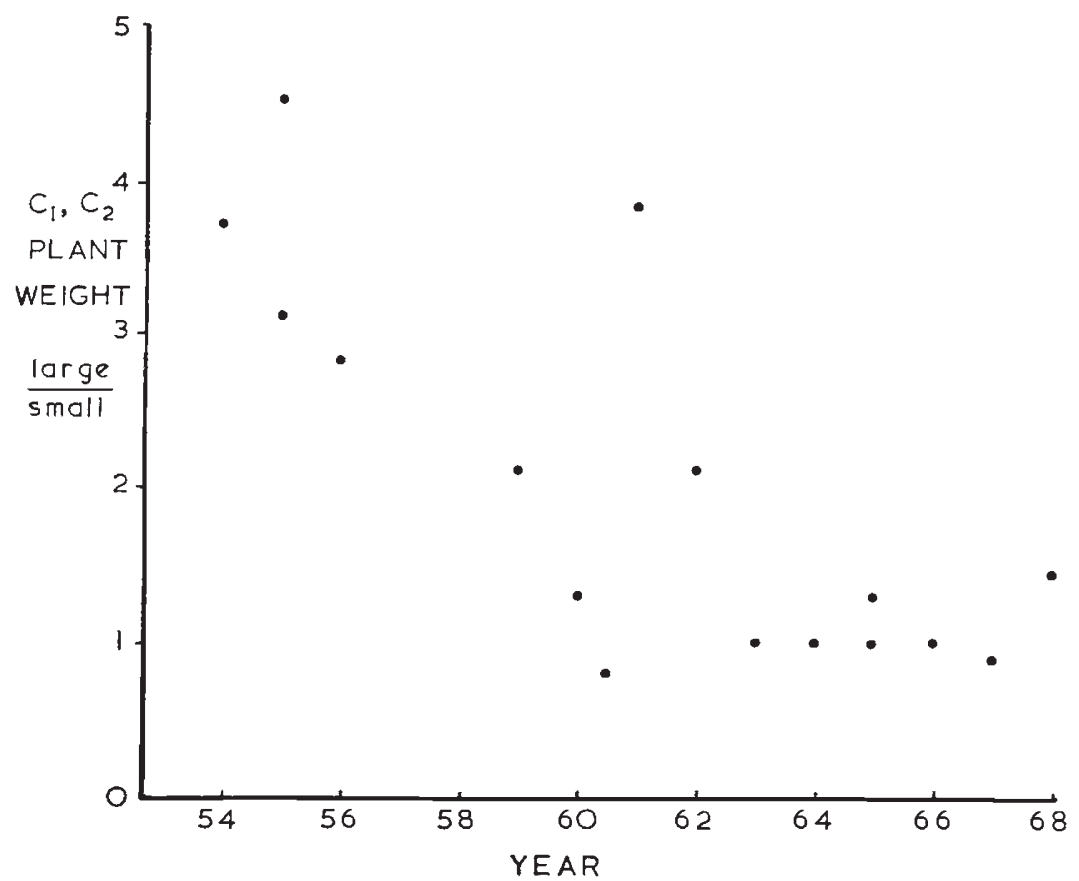

FIG. 2.-Ratio given by the mean weight of the larger plants resulting from induction in one environment divided by the mean weight of the smaller plants from induction in another environment, plotted against the year in which the inducing environments were applied.

\section{ThE ENVIRONMENT}

(i) General and specific inducing environments

The environments used for inducing heritable changes are not very different from natural environments, nor environments used in cultivation, except that there is normally some imbalance of nutrients and greenhouse temperatures are required for at least the first five weeks. The inducing environment is made up of a general component and a specific component. The specific environmental component is the specific fertiliser, or fertiliser combination, or other nutrient which, with the general component, induces either $\mathrm{L}$ or $\mathrm{S}$. The general environmental component comprises all environmental conditions or factors other than the specific components. One prerequisite for the induction of $\mathrm{L}$ or $\mathrm{S}$ genotroph is that the general component of the inducing environment must be capable of producing rapidly growing, healthy plants. This normally means a fertile soil, sufficient light and greenhouse temperatures during at least the first five weeks of growth. 
Another prerequisite is that the specific component of the inducing environment must not give stunted or unhealthy looking plants although different specific components may result in plants varying greatly in size. Environments for inducing other changes not dealt with in this paper do not necessarily have these restrictions.

\section{(ii) $\mathrm{n}$ and $\mathrm{p}$ as specific environmental components}

In experiments previously described Pl plants were grown for the first five weeks in a heated greenhouse and then transplanted into the field. In the greenhouse they were grown in boxes containing compost made up from soil from the field, peat and granite chippings, to which the fertilisers were added in solution. In the field they were grown in plots to which the appropriate fertilisers had been applied. Table $\mathrm{l}$ shows the induced changes

TABLE 1

Mean plant weights $(g$.$) of \mathrm{C}_{1}$ and $\mathrm{C}_{2}$ generations of $\mathrm{C}_{0}$ plants grown in different combinations of fertilisers in 1954

$\begin{array}{ccccccccc}\text { Fertilisers } & n p k & n p & n k & n & p k & p & k & (i) \\ C_{1} & 63 & 54 & 21 & 69 & 64 & 47 & 45 & 34 \\ C_{2} & 99 & 90 & 37 & 106 & 88 & 87 & 70 & 46\end{array}$

appearing in the first generation, $C_{1}$, of parents, $C_{0}$, treated in 1954. They range from about 21 grams for $C_{1} n k$ to about 65 grams for $C_{1} n p k, C_{1} n$ and $C_{1} p k$. The large $C_{1} n p k$, and the small $C_{1} n k$, types have been extensively used in further studies and are the $\mathrm{L}$ and $\mathrm{S}$ stable genotrophs appearing in more recent reports.

It is a simple matter to repeat the specific components of the environment during the first five weeks of growth since this involves no more than measuring out solutions. It is impossible to maintain the same general environmental component each year in the greenhouse or in the field, nor the specific component in the field since an assessment has to be made of the fertiliser requirements of each plot. For example $C_{1} p$ in table $\mathrm{I}$ is intermediate and is plastic, but when it was grown with phosphorus fertiliser in subsequent generations it rapidly changed to a small stable type comparable with $C_{1} n k$. Independent experiments showed that this was not due to an accumulative effect but that the $p \mathrm{H}$ of the soil had dropped and with low $p \mathrm{H} p$ fertiliser induces immediately the small stable genotroph $\mathrm{S}$.

These and other experiments led to the conclusion that the most reliable specific environmental components for inducing the large stable genotroph $(\mathrm{L})$ and the small stable genotroph (S) over a range of general environmental components were $n$ fertiliser with average $p \mathrm{H}$ to induce $\mathrm{L}$, and $p$ fertiliser with low $p \mathrm{H}$ to induce $\mathrm{S}$. This was further borne out by nuclear DNA analysis. Initial studies on $\mathrm{L}$ descended from $C_{1} n p k$ and on $\mathrm{S}$ descended from $C_{1} n k$ showed that $\mathrm{L}$ had 16 per cent. more nuclear DNA than S. Subsequent studies on $\mathrm{L}$ induced by $n$ and $\mathrm{S}$ induced by $p$ gave precisely the same results, the DNA difference being obtained wherever $n$ and $p$ fertilisers were used. Reciprocal crosses between $n k$-induced and $p$-induced plants indicated they were genetically identical in plant weight; table 2 shows there is no complementation and all are less than one-third the size of the $\mathrm{L}$ genotroph which was grown for comparison. 
(iii) The general component of the inducing environment

When it was clear that the specific environments of $n$ and $p$ were suitable for inducing $\mathrm{L}$ and $\mathrm{S}$, further changes were made to obtain better control of the general environmental component. For this, and other reasons, instead of transplanting the parental plants receiving the inducing treatments into the field they were grown from sowing to maturity in 5-inch pots in the greenhouse. The field plots were, however, used for the $C_{1}$ and later generations since $\mathrm{L}$ and $\mathrm{S}$ genotrophs once induced should be stable and unaffected by year-to-year variations in the field. At the same time these environmental studies were scaled down to allow room for experiments on the genetic aspects of induction which were dependent for their success on inducing

TABLE 2

Mean plant weigths (g.) of $S$ genotroph plants, induced respectively by $\mathrm{nk}$ and $\mathrm{p}$ fertilisers, and their reciprocal crosses compared with the $L$ genotroph induced by $\mathrm{npk}$

\begin{tabular}{|c|c|c|c|}
\hline$n k$ & $n k \times p$ & $p \times n k$ & $p$ \\
\hline 12 & 11 & 13 & 10 \\
\hline
\end{tabular}

heritable changes. Figure 2 shows, however, that in these later experiments the effectiveness of $n$ and $p$ for inducing $\mathrm{L}$ and $\mathrm{S}$ disappears. Assuming that the plants of the P1 stock are no different from those previously used, this means that the newly imposed general environmental conditions inhibit induction, or if not these, other factors of the general environment whose influences on induction are unknown, or are difficult or impossible to control from year to year. The following items, though obvious and mundane, are real practical problems; temperature, particularly in the first few weeks of induction normally in April; greenhouse heating including changes from boiler to electric heating; sunshine; watering and greenhouse ventilation; insecticide and fungicide applications; soil from the field used in making of compost; daylength changes due to neighbouring artificial lighting; change of gardeners; etc. Environments are most easily controlled and repeated using culture solutions in controlled environment growth rooms, and I am grateful to Professor Anton Lang for permitting me the use of these facilities at the Earhart Laboratories, California Institute of Technology, for carrying out the following experiments giving more information on the general environmental component.

\section{(iv) Induction experiments with culture solutions in the Earhart Laboratories}

Seed from a sample of the $\mathrm{Pl}$ genotroph, designated $\mathrm{Pl} x$, was sent to the Earhart Laboratories, California Institute of Technology, in 1961, where, with the co-operation of Professor Lang and his staff, they were sown in cups containing a mixture of gravel and vermiculite and washed with one-tenth strength Hoagland's solution daily. They were sown in June and grown in a 16-hour day, 8-hour night, and at a temperature of $19^{\circ} \mathrm{C}$. for 8 hours during the day and $11^{\circ} \mathrm{C}$. for the remaining 16 hours (19/11 regime). Seed was collected from the plants in November and designated Ply. Seed from the $\mathrm{Pl} x$ sample was also brought over later and both $\mathrm{Pl} x$ and $\mathrm{Pl} y$ seed were used in an environmental induction experiment at Earhart. 
In April of the same year seed from the $\mathrm{Pl} x$ sample was sown in an induction experiment at Aberystwyth using $n$ and $p$ treatments, the details of which are given below. The experiment therefore has three parts as shown in table 4; one at Aberystwyth using $\mathrm{Pl} x$ seed, designated $\mathrm{Pl} x A$; one at Earhart using $\mathrm{Pl} x$ seed, $\mathrm{Pl} x E$; and another at Earhart using $\mathrm{Pl} y$ seed, $\mathrm{Pl} y E$.

At Earhart $\mathrm{Pl} x$ and $\mathrm{Pl} y$ were given the same set of treatments. Three plants for each treatment were grown in half-pint pots, one plant per pot, which were filled with the standard mixture of gravel and vermiculite, and allowed free drainage through the base. The $n, p$ and $H o$ treatments are given in table 3 . The plants were sown on 7 th November 1961, and harvested 25th March 1962. They all received 16-hour day, 8-hour night, but

TABLE 3

$\mathcal{N}$ utrient regimes applied to the $\mathrm{C}_{0}$ generation in culture solutions at Earhart

\begin{tabular}{lll} 
& \multicolumn{2}{c}{$n$ treatment } \\
\cline { 2 - 3 } Sun. & Water & 4 p.m. \\
Mon. & $n$ & Woter \\
Tues. & $n$ & Water \\
Wed. & $H o$ & Water \\
Thurs. & $n$ & Ho \\
Fri. & $n$ & Water \\
Sat. & Water & Water
\end{tabular}

$n$ treatment (above); $n$ is 0.05 per cent. ammonium sulphate

$H o$ is $\frac{1}{5}$ strength Hoagland's solution.

$p$ treatment; the same regime as above except that 0.2 per cent. triple superphosphate replaces $n$.

Ho treatment (see table 4); $\frac{1}{2}$ strength Hoagland's solution every morning, water in afternoon.

Water and solutions applied to saturation with subsequent free drainage.

there were two temperature regimes (table 4), some receiving the 19/11 regime previously mentioned and the others receiving $27 / 15$, i.e. $27^{\circ} \mathrm{C}$. for 8 hours during the day and $15^{\circ} \mathrm{C}$. for the remaining 16 hours. This is the parental $\left(C_{0}\right)$ generation.

Seed collected from each set of three plants of each $C_{0}$ treatment shown in table 4 was mixed and sown on 5th April 1962. This is the first $\left(C_{1}\right)$ generation. Four $C_{1}$ plants were grown in each combination and the general procedure was the same as for $C_{0}$ except that all the plants were grown in the 27/15 temperature regime and the nutrients were switched round, those which received $H_{0}$ in $C_{0}$ now received $n$ or $p$, and those which received $n$ or $p$ in $C_{0}$ received $H o$. The plants were harvested in early July 1962 , and seed from the four plants of each combination mixed and used for tests on the $C_{2}$ and later generations carried out at Aberystwyth.

For the third part of the experiment, grown entirely at Aberystwyth, the earlier type of induction procedure was used. In the parental $\left(C_{0}\right)$ generation $n$ and $p$ were applied separately to soil compost and the plants were transplanted out after five weeks in the greenhouse. There were five $\mathrm{Pl} x$ plants for each of the two treatments. Seed from two $C_{0}$ plants receiving the $n$ treatment was mixed to give one $n$ sample, and seed from two other $n$ 


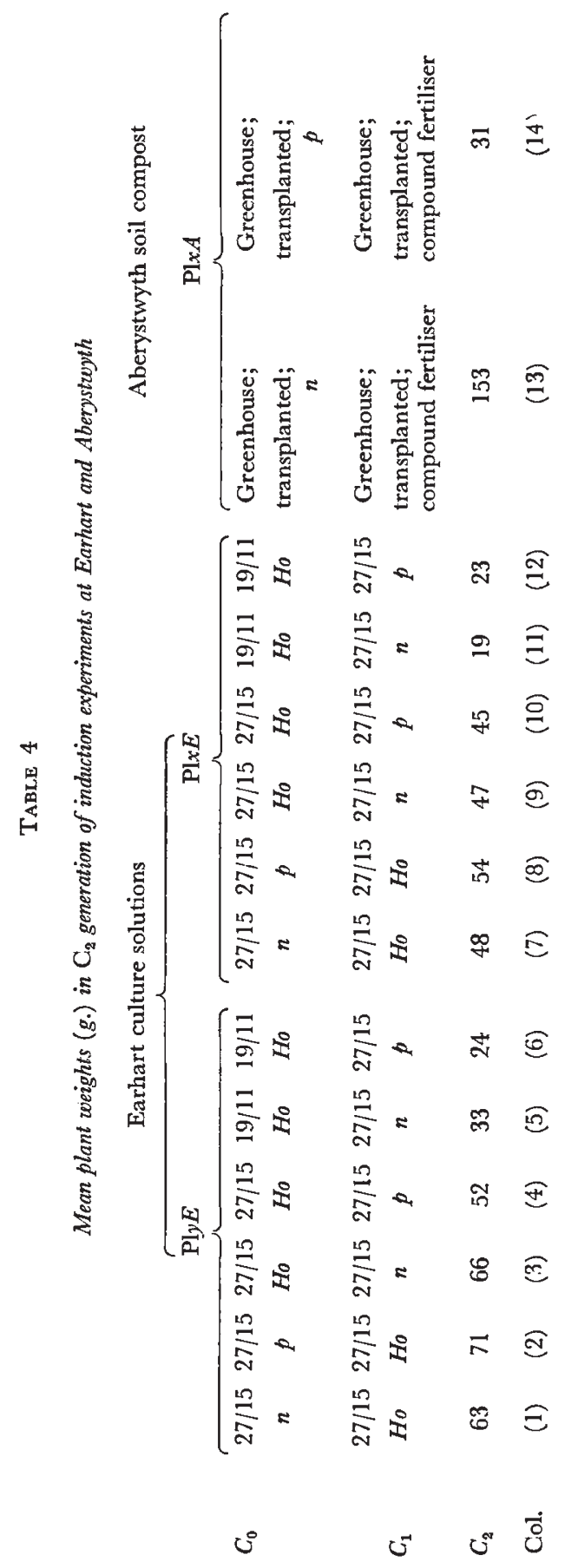


plants to give a second $n$ sample. Similarly, two $p$ samples were obtained. Ten plants were grown in 1963 from each of the four samples to give the first generation $\left(C_{1}\right)$. They were grown for the first five weeks in a greenhouse with John Innes base compost and transplanted into field plots which had received a compound fertiliser. Seed from each set of ten plants was used for tests on the $C_{2}$ and later generations.

Mean plant weights for the $C_{2}$ generation are given in table 4 . They were all grown for the first five weeks in the greenhouse and transplanted into the field. The $C_{2}$ of $\mathrm{Pl} x A$ was grown in 1964; the $C_{2}$ generations of $\mathrm{Pl} x E$ and $\mathrm{Pl} y E$ were grown in 1963 in separate experiments so that the overall means of $\mathrm{Pl} x A, \mathrm{Pl} x E$ and $\mathrm{Pl} y E$ are not comparable. In the induction experiment carried out solely at Aberystwyth, $\mathrm{Pl} x A$, there is a highly significant difference between the progeny of $n$ - and $p$-treated plants: $C_{2} n$ is five times the size of $C_{2} p$ (columns (13) and (14)) and in appearance they are similar to $\mathrm{L}$ and $\mathrm{S}$ genotrophs previously induced. Later generations have confirmed that they are stable $\mathbf{L}$ and $\mathrm{S}$ genotrophs.

In the $C_{2}$ generation of plants treated in culture solutions at Earhart, however, there is no evidence whatever of $n$ and $p$ inducing heritable changes (compare columns (1) and (2), (7) and (8)). In fact, $C_{2} p$ is slightly larger than $C_{2} n$, though not significantly so. A check is given by the progeny of the $C_{1}$ plants treated with $n$ and $p$, columns (3) (4), (5) (6), (9) (10), (11) (12), none of which gives a significant difference, although $n$ does give a slightly larger plant than $p$ possibly due to maternal effects. There is instead a dierence due to temperature. Plants whose grandparents were grown with $H o$ at 27/15 are nearly twice the size of plants whose grandparents were grown with $H o$ at $19 / 11$. The differences are significant $(\mathrm{P}<0 \cdot 1$ per cent.) and they are given by four independent sets of Co plants, i.e. columns (3) (5), (4) (6), (9) (11), (10) (12). Plants whose grandparents received $n$ or $p$ also give approximately the same weights as the 27/15 Ho groups. Consequently the nutrients $n, p$ and $H o$ do not induce any changes whereas there is an induced difference due to the temperatures $27 / 15$ and $19 / 11$.

Table $5(a-\ell)$ summarises the results of tests made at Aberystwyth in later years on the progeny of plants from the $19 / 11$ and $27 / 15$ environments. $(a),(b),(c)$ and $(d)$ show that the differences between the 27/15 and 19/11 types persist into the $C_{3}$ and $C_{4}$ generations; $(e)$ and $(d)$ show that 27/15 plants have the same weight as $\mathrm{Pl}$ but $19 / 11$ plants are half the size; $(a)$ and (b) show that 27/15 $\mathrm{Pl} y E$ and $\mathrm{Pl} x E$ are the same, and $19 / 11 \mathrm{Pl} y E$ and $\mathrm{Pl} x E$ are the same; (e) shows that reciprocal crosses between 27/15 and 19/11 types give equilinear inheritance. The conclusions are: (i) Since only the 19/11 type is different from $\mathrm{Pl}$ this temperature regime induced a change, but not $27 / 15$. (ii) $19 / 11$ regime induced this change from November to March with half-strength Hoagland's, but the 19/11 regime with one-tenth strength Hoaglands from May to November did not induce any change since $\mathrm{Pl} y E$ progeny are the same as $\mathrm{Pl} x E$; the season as well as nutrients may be responsible because although the ambient temperature was controlled at 19/11 the amount of light in the greenhouse was not. (iii) Equilinear inheritance implicates the nucleus (dominance, given by both reciprocals often occurs in crosses in between genotrophs, discussed in section 4). The $19 / 11$ induced type is not the same as the $\mathrm{S}$ genotroph. Although it approximates to $\mathrm{S}$ in weight it is taller, like $\mathrm{Pl}$, and presumably arises from a different induced change from that which gives rise to $\mathrm{L}$ or $\mathrm{S}$ genotroph. 
The significance of the Earhart experiments lies, however, not so much in the induction of the 19/11 type but more generally in the failure to obtain induced changes in the culture solutions and Earhart environments. A number of other experiments carried out in the Earhart Laboratories in collaboration with Professor Lang also showed that a large range of other environments is ineffective for induction. The nitrogen-treated plants at Earhart were dark green compared with the phosphorus-treated plants, and

\section{TABLE 5}

Tests on the $\mathrm{C}_{3}$ and $\mathrm{C}_{4}$ generations of $27 / 15$ and $19 / 11$ temperature treatments at Earhart. The column numbers refer to those of table 4. Mean plant weights (grams)

(a) $C_{3}$ generation 1964

\begin{tabular}{|c|c|c|c|c|}
\hline \multirow[b]{2}{*}{ Origin } & \multicolumn{2}{|c|}{$27 / 15$} & \multicolumn{2}{|c|}{$19 / 11$} \\
\hline & Col. & Col. & Col. & Col. \\
\hline $\begin{array}{l}\mathrm{Pl} y E \\
\mathrm{Pl} x E\end{array}$ & $\begin{array}{l}\text { (3) } 46 \\
\text { (9) } 44\end{array}$ & $\begin{array}{r}\text { (4) } 10 \\
\text { (10) } 41\end{array}$ & $\begin{array}{r}\text { (5) } 24 \\
\text { (11) } 23\end{array}$ & $\begin{array}{r}\text { (6) } 25 \\
\text { (12) } 25\end{array}$ \\
\hline
\end{tabular}

(b) Analysis of variance of $(a)$

$\begin{array}{lrcc}\quad \text { Item } & \text { d.f. } & \text { Mean square } & \text { Probability } \\ \text { Replicates } & 3 & 4 \cdot 7 & - \\ \text { Pl } x \text {-Pl } y & 1 & 38.3 & - \\ 19 / 11-27 / 15 & 1 & 861 \cdot 1 & <0 \cdot 001 \\ n-p & 1 & 10 \cdot 1 & - \\ \text { Interactions } & 4 & 11.7 & - \\ \text { Error } & 21 & 28.4 & -\end{array}$

(c) $C_{3}$ generation, 1964, of $\mathrm{Pl} x E, n$ and $p$ treatments summed

$\begin{array}{ccc}27 / 15 & 19 / 11 & \mathrm{Pl} x \text { (once grown) } \\ 43 & 22 & 45\end{array}$

(d) $C_{4}$ generation, 1966, of $\mathrm{Pl} x E$

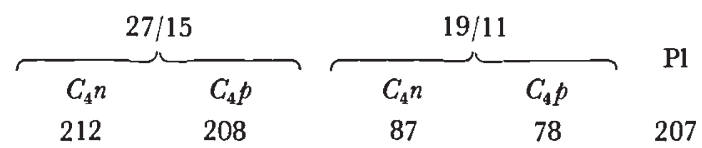

(e) $\mathrm{F}_{1}$ of crossings between $27 / 15$ and $19 / 11$ types from $n$ treatments. $\mathrm{Pl} y E$ and $\mathrm{Pl} x E$ are separate experiments grown in 1964

$\begin{array}{lcccc}\text { Origin } & 27 / 15 & 27 / 15 \times 19 / 11 & 19 / 11 \times 27 / 15 & 19 / 11 \\ \mathrm{Pl} y E & 45 & 46 & 43 & 22 \\ \mathrm{Pl} x E & 29 & 28 & 26 & 18\end{array}$

these nutrients were clearly having an affect on the growth of the treated parental plants. Consequently the general inducing environment at Earhart must have been unsuitable for induction, in common with the later inducing experiments at Aberystwyth (fig. 2) where $n$ and $p$ were applied to Pl plants grown to maturity in 5-inch pots with compost. The treated parental plants in culture solutions at Earhart and in pots at Aberystwyth were one-third to one-fifth the size of treated parental plants transplanted into the field in the earlier experiments. This implies that given the specific inducing environments all that is required of the general inducing environment is that it promotes rapid, healthy growth. 


\section{The GeNOTYPe}

\section{(i) Maintaining the plastic genotroph}

Because heritable changes can be induced in the flax variety Stormont Cirrus it is called a plastic genotroph. Other varieties of Linum ussitatissimum are not necessarily plastic and it is supposed that genetic differences between flax varieties, and between flax and linseed varieties, determine whether the variety is plastic or not. $\mathrm{L}$ and $\mathrm{S}$ are the large and small genotrophs induced from $\mathrm{Pl}$ by the environment but, although descended from $\mathrm{Pl}$, they are no longer plastic because no further major changes in plant weight in either direction can be induced in them. The genotrophic difference is to all appearances a genotypic difference. Therefore the possession of the plastic characteristic is dependent on the genotype of the plant and on its ancestral environments.

Seed of Stormont Cirrus was sent to this department on two occasions, in 1952 and 1957, from Stormont, Northern Ireland, and both samples proved to be plastic. In multiplying up the $\mathrm{Pl}$ stocks at Aberystwyth care had obviously to be taken to grow the Pl plants in environments which would maintain their plasticity, and in particular not in those environments which were at all similar to those known to induce the $\mathrm{L}$ and $\mathrm{S}$ stable genotrophs. Before starting the first induction experiment in 1954 (Durrant, 1962a), the seed of Stormont Cirrus was sown in 1953 in boxes out of doors in soil (not made up into compost) and transplanted into field plots which had not recently received fertilisers. It was supposed that this would ensure an average balanced type of environment, and that the seed taken from a limited number of plants would be suitable for the environmental induction experiments the following year.

Although this proved satisfactory, with increased knowledge of the environments which induce the stable $\mathrm{L}$ and $\mathrm{S}$ genotrophs, and because of the possibility of accumulating small changes over generations, further precautions were taken in multiplying up $\mathrm{Pl}$ seed, primarily to ensure that the soil was not too fertile. About $20 \mathrm{Pl}$ plants were grown per 7-inch pot containing compost made up with soil from the field with a small measured quantity of fertiliser added, the pots being kept out of doors all the time except for ripening off the seed already set in the greenhouse. There was no apparent change in the appearance of these plants over the years. Their plant weight relative to the weights of $L$ and $S$, their interaction with the environment, nuclear DNA content and the $\mathrm{H}$-h capsule character were all unchanged, and it was assumed that this environment was suitable for maintaining the plastic type.

On the other hand it is probable that the induction of $\mathrm{L}$ or $\mathrm{S}$ is more than a single event. General observations and nuclear DNA studies show that the first five weeks of growth of $\mathrm{Pl}$ is an important period for induction. At the sixth week flower primordia are formed and the subsequent developmental phase could have a different environmental requirement for induction. Consequently the multiplication environment could induce in $\mathrm{Pl}$ a heritable change restricted to a loss of plasticity unaccompanied by a phenotypic change to L or S, and the 19/1 I type of plant induced at Earhart might be intermediate between this and a full change to $\mathrm{S}$. The following experiment supplies indirect evidence of induced changes which are potentially heritable 
but which do not give heritable phenotypic changes in the treated plants themselves.

\section{(ii) Transmission of induced change by crossing}

This experiment was designed to test whether $\mathrm{L}$ and $\mathrm{S}$, although stable and showing no induced change themselves, were capable of transmitting an induced change by growing them in inducing environments and at the same time crossing them to $\mathrm{Pl}$ grown in a non-inducing environment. In 1960 crossings were made between $\mathrm{L}, \mathrm{S}$ and $\mathrm{Pl}$ plants growing in $n, p$ and control environments. Not all possible crosses were made and those to be discussed are numbered (1) to (7) in table 6 . The seed was sown in boxes of compost receiving the $n p k$ and $p$ fertilisers and the plants transplanted later into field plots receiving the respective fertilisers. Because of lack of space the boxes were put into the greenhouse two or three at a time for a week or so and left outside for the remainder of the four-week period while the others took their turn. The full inducing environment in the first five weeks of growth was consequently not given, otherwise they were similar to those used in the earlier induction experiments. The control environment in which some of the Pl plants were grown consisted of 7-inch pots containing compost with quarter the usual quantity of John Innes base fertiliser added. Four Pl plants were grown in each pot and they remained outside in the pots from sowing to maturity.

The $C_{1}$ generation (which is also the $\mathrm{F}_{1}$ of the crosses) was not grown until 1963 and the age of the seed, 3 years, may have aggravated maternal effects which tend to be more pronounced in flax seed obtained from crossing, particularly if the weather conditions during crossing are not good. The $C_{1}$ plants, approximately 20 for each cross, were forced in a heated greenhouse in compost with J.I. base fertiliser for five weeks and transplanted with single plant randomisation into the field which had received a compound fertiliser.

The parental environments were as follows. In crosses of $\mathrm{L} \times \mathrm{L}, \mathrm{S} \times \mathrm{S}$, $\mathrm{Pl} \times \mathrm{Pl}$, both parents were grown either in $n p k$ or $p$; in crosses $\mathrm{L} \times \mathrm{Pl}$ and $\mathrm{Pl} \times \mathrm{L}$, the $\mathrm{Pl}$ parents were grown in control only and the $\mathrm{L}$ parents were grown in $n p k$ or $p$; in crosses $\mathrm{S} \times \mathrm{Pl}$ and $\mathrm{Pl} \times \mathrm{S}$ the $\mathrm{Pl}$ parents were grown in control only and the $\mathrm{S}$ parents in $n p k$ or $p$. The $C_{1}$ plant weights are given in table 6.

Averaging over $n p k$ and $p$, the seven pairs of crosses give values more or less as expected. $\mathrm{L} \times \mathrm{L}$ is six to nine times the weight of $\mathrm{S} \times \mathrm{S}: \mathrm{Pl} \times \mathrm{Pl}$ is nearer $\mathrm{L} \times \mathrm{L}$ than $\mathrm{S} \times \mathrm{S}$, which occurs in some environments (section 4 ) and is not necessarily an induced change. $\mathrm{S} \times \mathrm{Pl}$ and $\mathrm{Pl} \times \mathrm{S}$ have values between $\mathrm{Pl} \times \mathrm{Pl}$ and $\mathrm{S} \times \mathrm{S}: \mathrm{L} \times \mathrm{Pl}$ and $\mathrm{Pl} \times \mathrm{L}$ have values on average between $\mathrm{Pl} \times \mathrm{Pl}$ and $\mathrm{L} \times \mathrm{L}$.

Induced changes are expected in the $\mathrm{Pl} \times \mathrm{Pl}$ cross only since in all the other crosses only the stable genotrophs, $\mathrm{L}$ or $\mathrm{S}$, were grown in $n p k$ and $p$, the Pl plants to which they were crossed being in the control environment. In $\mathrm{Pl} \times \mathrm{Pl}$ the difference between $n p k$ and $p$ is hardly significant at the 5 per cent. level but significant on a one tail test since the difference is in the expected direction. The full induced changes have not occurred presumably because the full inducing environments were not given. No changes have occurred in the $\mathrm{S} \times \mathrm{S}$ cross, but in $\mathrm{L} \times \mathrm{L}$ and in each of the four remaining crosses there is a significant difference, $p$ parental environment giving larger 
plants than the $n p k$ parental environment. In the $\mathrm{L} \times \mathrm{L}$ cross this could be maternal, due to a carry-over effect of the fertilisers, but not in the $\mathrm{PI} \times \mathrm{L}$ and $\mathrm{Pl} \times \mathrm{S}$ crosses, where only the male parents received the $n p k$ and $p$ fertilisers, and since there are no maternal effects in $\mathrm{S} \times \mathrm{S}$ it is less likely to have occurred in $\mathrm{S} \times \mathrm{PI}$.

Crosses (4)-(7) between other combinations of fertilisers showed no distinctive patterns and only rarely a significant difference at the 5 per cent. level and the overall mean plant weight for each cross is entered with the rest of the $C_{1}$ data in table 6 . These mean plant weights are similar to those given by the $p$ parental environment and therefore the differences in the $C_{1}$ generation are due to the $n p k$ parental environment inducing a smaller

TABLE 6

Mean plant weights (grams) in the $\mathrm{C}_{1}$ and $\mathrm{C}_{2}$ generations of crosses between $L, S$ and Pl parent plants grown in different environments. Crosses (1)-(3); both male and female parents of each cross grown in $\mathrm{npk}$, or $\mathrm{P}$, shown at column heads. Crosses $(4)-(7)$; male and female $P l$ parents grown in control environment throughout; male and female $L$ and $S$ parents grown in $\mathrm{npk}$, or $\mathrm{p}$, shown at column heads. Mean plant weights from other combinations given for comparison. Standard error of $\mathrm{C}_{1}$ mean plant weights $=3 \cdot 14$

\begin{tabular}{|c|c|c|c|c|c|c|c|}
\hline \multirow[b]{2}{*}{ Cross } & \multicolumn{2}{|c|}{$C_{1}$} & \multirow{2}{*}{$\begin{array}{c}\text { Other } \\
\text { combinations }\end{array}$} & \multicolumn{2}{|c|}{$C_{2} 1964$} & \multicolumn{2}{|c|}{$C_{2} 1965$} \\
\hline & $n p k$ & $p$ & & $n p k$ & $p$ & $n p k$ & $p$ \\
\hline (1) $\mathrm{L} \times \mathrm{L}$ & $37 \cdot 9$ & $57 \cdot 6$ & - & $44 \cdot 8$ & $40 \cdot 0$ & - & - \\
\hline (2) $\mathrm{S} \times \mathrm{S}$ & $6 \cdot 6$ & 6.7 & - & $15 \cdot 2$ & 14.0 & - & - \\
\hline 3) $\mathrm{Pl} \times \mathrm{Pl}$ & $42 \cdot 0$ & $34 \cdot 6$ & - & - & - & - & - \\
\hline (4) $\mathrm{L} \times \mathrm{Pl}$ & $37 \cdot 7$ & $48 \cdot 4$ & $48 \cdot 7$ & $25 \cdot 4$ & $35 \cdot 4$ & $50 \cdot 6$ & $64 \cdot 1$ \\
\hline 5) $\mathrm{Pl} \times \mathrm{L}$ & $32 \cdot 3$ & 41.5 & $46 \cdot 2$ & $29 \cdot 2$ & $32 \cdot 4$ & $38 \cdot 4$ & $60 \cdot 8$ \\
\hline 6) $\mathrm{S} \times \mathrm{P} 1$ & $15 \cdot 7$ & $28 \cdot 3$ & $27 \cdot 0$ & $22 \cdot 7$ & $30 \cdot 1$ & - & - \\
\hline 7) $\mathrm{Pl} \times \mathrm{S}$ & $17 \cdot 5$ & $31 \cdot 8$ & $29 \cdot 0$ & $21 \cdot 8$ & $20 \cdot 2$ & - & - \\
\hline
\end{tabular}

plant in the crosses $\mathrm{L} \times \mathrm{PI}, \mathrm{PI} \times \mathrm{L}, \mathrm{S} \times \mathrm{PI}, \mathrm{PI} \times \mathrm{S}$. The mean plant weights in table 6 of some $C_{2}$ plants grown in 1964 show no parental environment effect in the $\mathrm{L} \times \mathrm{L}$ nor $\mathrm{S} \times \mathrm{S}$ cross, and where it occurred in the $C_{1}$ it was presumably maternal, or a transient induced change. In the other four crosses grown in 1964 the $\mathrm{L}$ and $\mathrm{S}$ parental environments effect has reappeared, although it is reduced a little overall. This reduction is probably due to interaction with the 1964 environment for the differences due to parental environment are even larger in the $C_{2}$ generation of the two crosses grown in 1965.

These induced changes in plant weight are paralleled by changes at the $h$ locus (Durrant and Nicholas, 1970). PI and S are $H H$ (hairy septa) and $\mathrm{L}$ is $h h$ (hairless). In the $n p k$ parental environment the crosses $\mathrm{L} \times \mathrm{PI}$ and $\mathrm{PI} \times \mathrm{L}(h h \times H H, H H \times h h)$ gave $10 H$ and $38 h$ plants in the $C_{1}$, which should all be phenotypically $H$. Also in the $n p k$ parental environment the crosses $\mathrm{S} \times \mathrm{Pl}$ and $\mathrm{Pl} \times \mathrm{S}(H H \times H H)$ gave all $H$ phenotype in the $C_{1}$, but $28 H$ and $10 \mathrm{~h}$ in the $C_{2}$ which should all be homozygous $H H$. No anomalous ratios of this type occurred in the $C_{1}$ or $C_{2}$ of the same crosses in the parental environment, nor in any of the other environmental combinations. This is additional evidence for the association of induced changes at the $h$ locus with induced changes in factors determining plant weight.

The experiment shows that the $\mathrm{L}$ and $\mathrm{S}$ genotrophs, although phenotypically stable and showing no permanent induced changes themselves, are able to transmit some stimulus or change, induced by the environment, 
which brings about a permanent change when crossed with $\mathrm{Pl}$ grown in a control environment. The inversion of the effects of the $n p k$ and $p$ fertilisers may be a characteristic of their transmission by L and S. This implies there must be at least two events when $\mathrm{L}$ and $\mathrm{S}$ are successfully induced from $\mathrm{Pl}$ in one generation, the $\mathrm{Pl}$ genotroph and the environments at successive stages of development being in themselves sufficient for both to occur. The first event may be a transient induced change which occurs generally; the second event is less common and stabilises the initial change making it permanent in inheritance. This interpretation, however, sustains both general explanations of the pattern of induction shown in fig. 2 in that (i) the environments are critical and different ones may be required at different stages of development, (ii) the Pl genotroph may not have changed phenotypically in the multiplication environment but it could have changed genetically, i.e. genotrophically, in its ability to stabilise induced changes.

\section{Genotroph/environment interaction}

It was supposed that plants with a low developmental stability would be more likely to transmit environmental effects of one generation to the next. From comments in the literature on the importance of seed quality, and from general observations, flax appeared at the outset the most promising of the inbreeding crop plants for inducing at least semi-permanent heritable changes by the environment. On the other hand, the strong interactions with the environments, although interesting, cause difficulties when testing $C_{1}$ and $C_{2}$ generations for induced changes, and when carrying out genetic experiments with the genotrophs in the field. Seed obtained from crosses varies in quality and quantity with weather conditions, the environment in which the plants are growing, and the plants being crossed. With poor seed set, or poor germination, selection may have occurred and the experiments are normally abandoned. There is also concern when, as sometimes happens, large variation occurs within plots between plants which are supposed to be genetically (or genotrophically) identical. Is the variation due to developmental instability, a break-down of the genotrophs, genetic impurity, or heterozygosity? Several studies on these situations showed that genotroph/environment interaction, or developmental instability, is responsible. Three examples are given below. In the first two examples the plots were selected for analysis here because of the high variation within them; normally the variation is much less, typical examples of which have been given (Durrant, 1962a).

\section{(i) Variation in the Pl genotroph}

Some plants of the $\mathrm{Pl}$ genotroph were included in a randomised block field experiment with six replicates. Each block contained a plot of five Pl plants which were taken at random from boxes and transplanted into the field in somewhat dry conditions by two gardeners, A and B, who worked in the same positions relative to the positions of the five plants per plot. Because of the particularly high variation among the plants (table $7 a$ ) they were analysed in detail (table $7 b$ ). The overall differences between the five positions is almost entirely due to the difference between gardeners. Keeping the plots item, though not significant, separate for comparative purposes and combining the other three with the residual item, the components of variation 
show that despite the gardeners' contribution more than half the variation is due to random variation. This could be at least in part due to genetic or genotrophic differences, but tests similar to those described below showed that the plants were genetically and genotrophically uniform.

\section{TABLE $7 a$}

Weights (grams) of Pl plants grown in six plots of five plants each. Plants are transplanted by two gardeners in the order shown. Apart from the large random variation there is a significant difference between gardeners, the centre plant being on average intermediate in weight

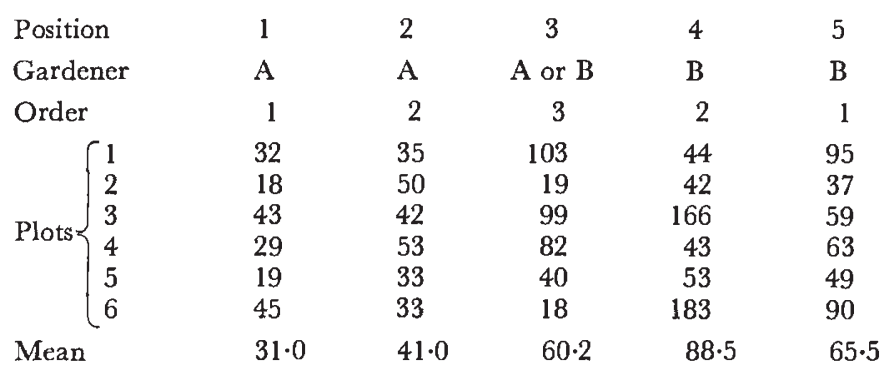

TABLE $7 b$

Analysis of variance

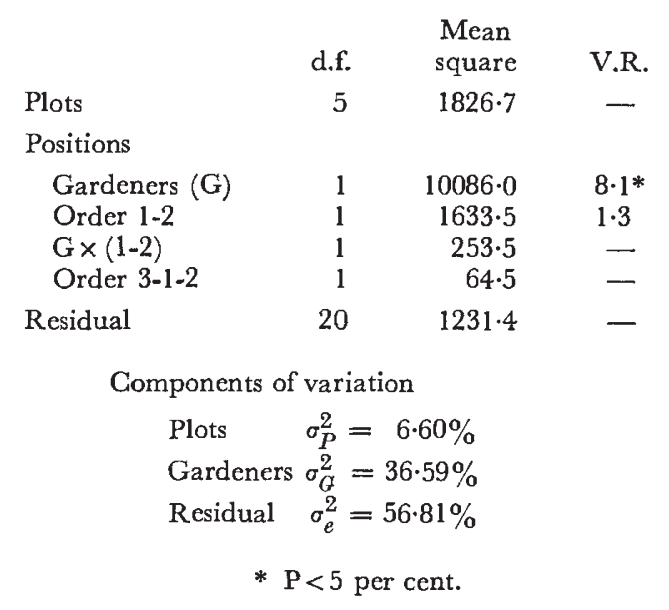

(ii) Variation within the $L$ genotroph

The L genotroph normally shows more between plant within plot variation than $\mathrm{S}$ or $\mathrm{Pl}$, although its coefficient of variation, correcting for its greater plant weight, is usually less than that of S. Two independent selection lines for large plant weight, and two for small plant weight, were begun in 1962 within $\mathrm{L}$ and continued for four generations. In the fourth generation four plots were chosen, one from each of the selection lines, containing the most within plot variation, and plants from seed collected from each of the five plants in each of the four plots were grown the following year in a randomised block field experiment with five replications. Five plants descended from each of the 20 parental plants were grown in each of the five replicates, giving 400 plants in all. 
The parental weights, the mean plant weights of the 20 offspring families and the analysis of variance are given in tables $8 a$ and $8 b$. The differences between the means of the selection lines on both parental and offspring

TABLE $8 a$

Weights (grams) of plants (parents) in four selection lines and mean plant weights of families (progeny) grown from them

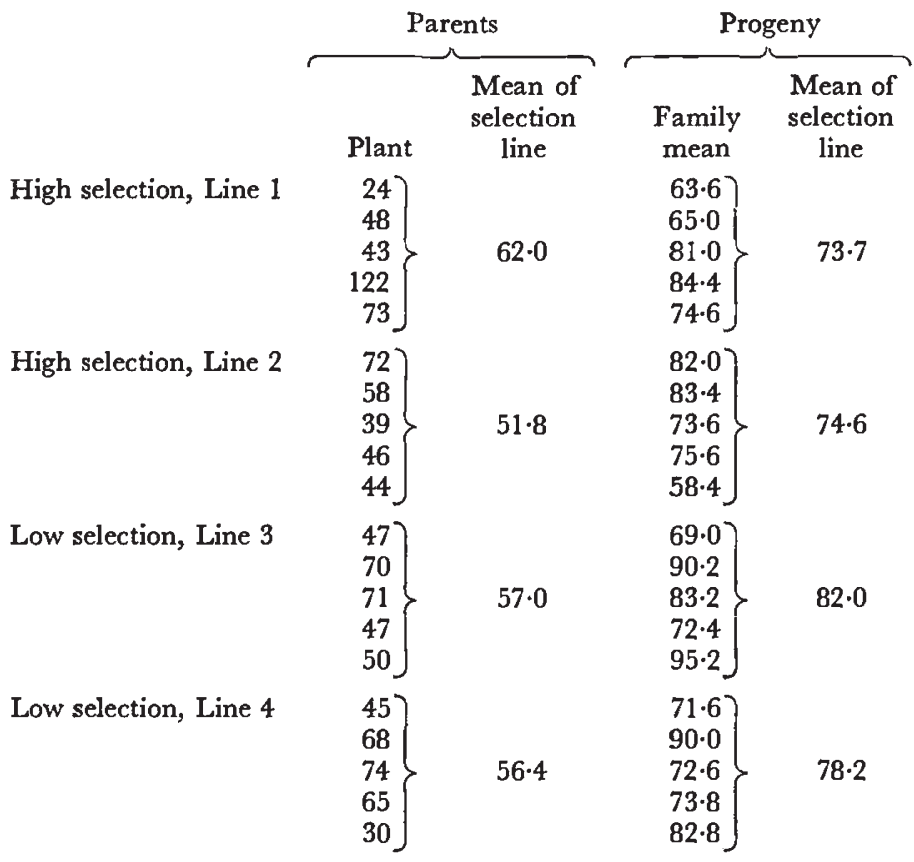

TABLE $8 b$

Analysis of variance of progeny, and regression on to parental values

\begin{tabular}{|c|c|c|c|}
\hline & d.f. & $\begin{array}{l}\text { Mean } \\
\text { square }\end{array}$ & V.R. \\
\hline $\begin{array}{l}\text { Families } \\
\text { Regression } \\
\text { Residual }\end{array}$ & $\begin{array}{r}19 \\
1 \\
18\end{array}$ & $\begin{array}{r}451 \cdot 5 \\
1379 \cdot 6 \\
399 \cdot 9\end{array}$ & $\begin{array}{l}2 \cdot 11^{*} \\
6 \cdot 45^{*} \\
1 \cdot 87^{*}\end{array}$ \\
\hline $\begin{array}{c}\text { Between lines } \\
\text { Regression } \\
\text { Residual }\end{array}$ & $\begin{array}{l}3 \\
1 \\
2\end{array}$ & $\begin{array}{r}356 \cdot 7 \\
9 \cdot 8 \\
530 \cdot 2\end{array}$ & $\frac{1 \cdot 67}{2 \cdot 48}$ \\
\hline $\begin{array}{l}\text { Within lines } \\
\text { Regression } \\
\text { Residual }\end{array}$ & $\begin{array}{r}16 \\
1 \\
15\end{array}$ & $\begin{array}{r}469 \cdot 3 \\
1465 \cdot 1 \\
402 \cdot 9\end{array}$ & $\begin{array}{l}2 \cdot 19^{*} \\
6 \cdot 85^{*} \\
1 \cdot 88^{*}\end{array}$ \\
\hline Error & 76 & $214 \cdot 0$ & - \\
\hline
\end{tabular}

generations are small and selection over the years has been ineffective. There are significant differences between the 20 families $(P<5$ per cent.) when tested against the error of the experiment. The variation between the families has been split into two parts, one consisting of linear regression on to 
the parental values, and the other for variation about the regression line. Both are significant, and the regression (which is positive) is also significant on a one-tail test against the variation about the regression line. The remainder of the analysis shows that the inherited differences between the families reside within the selection lines, not between. If these differences were transmitted one further generation then selection should pick them up. Selection has had no effect for four generations and there is no reason to believe that this parental generation is different from any previous one, and therefore the differences between the offspring must be due to maternal inheritance or to transient, induced heritable changes, and the differences between the parent plants within the plots, from 24 to 122 grams in one case, to developmental instability, or solely due to the environment. A difference in parental plant weight of 10 grams gives a mean plant weight difference between offspring families of about $1 \cdot 8$ grams.

TABLE 9

Mean plant weights $(g$.$) and heights (\mathrm{cm}$.$) of L, S, P l$ and $F_{1}$ and $F_{2}$ of reciprocal crosses between $L$ and $S$ grown in summer and zointer conditions. N Nuclear $D \mathcal{N} A$ values of $L, S$ and $P l$ in arbitrary units. Data taken from different experiments

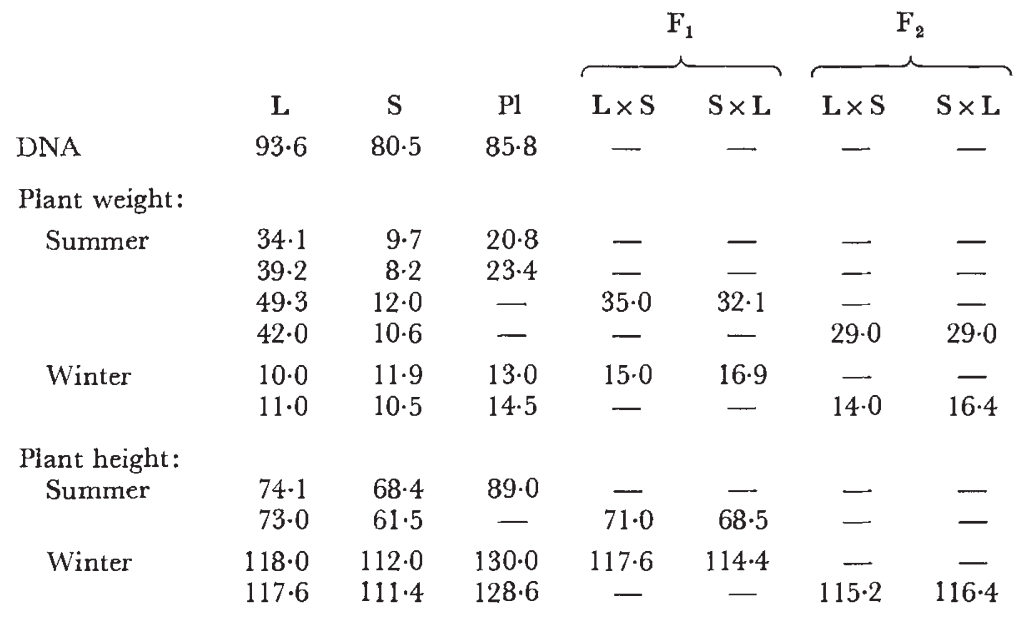

(iii) Symmetric and asymmetric induction

When the plants are grown under normal experimental conditions, i.e. in trays inside or outside an unheated greenhouse and transplanted into the field in summer, $\mathrm{Pl}$ is intermediate in weight between $\mathrm{L}$ and $\mathrm{S}$ (table 9). The induction of the plant weights of $\mathrm{L}$ and $\mathrm{S}$ is therefore symmetrical about P1. The induced changes in amount of nuclear DNA occurring at the same time are also symmetrical. $\mathrm{F}_{1}$ and $\mathrm{F}_{2}$ mean plant weights of the reciprocal crosses between $\mathrm{L}$ and $\mathrm{S}$ are intermediate (table 9), i.e. they show no dominance, as though the $\mathrm{Pl}$ genotroph is reconstituted on crossing $\mathrm{L}$ and $\mathrm{S}$.

When the plants are grown in a heated greenhouse in the winter with the addition of a small amount of artificial light, $\mathrm{L}$ and $\mathrm{S}$ have approximately the same plant weight whereas $\mathrm{Pl}$, and the $\mathrm{F}_{1}$ and $\mathrm{F}_{2}$, are larger than either of them. Therefore, (i) in this environment the induction of $\mathrm{L}$ and $\mathrm{S}$ may be judged to be asymmetric, and (ii) the $F_{1}$ and $F_{2}$ show heterosis. (iii) An 
extra generation grown in the winter gives a poor assessment of, for example, induction experiments of the previous summer. (iv) Pl responds to the change in the environment in the same way as the $F_{1}$ and $F_{2}$, which appear to reconstitute it, to give a heterotic effect. But $\mathrm{Pl}$ is presumed to be homozygous and although two gene models could be described they appear artificial and it is simpler to suggest that $\mathrm{Pl}$ and the $\mathrm{F}_{1}$ and $\mathrm{F}_{2}$ have an intermediate metabolism, or favourable genetic balance, which is relatively more efficient at high temperatures and lower light intensities. (v) If the relative amount of nuclear DNA is the same in the winter environment as in the summer, as appears to be the case, then amount of nuclear DNA is not always correlated with plant weight.

Plant heights are also given in table 9. $\mathrm{L}$ is consistently taller than $\mathrm{S}$, but $\mathrm{Pl}$ is significantly taller than either, in summer and winter, and plant height is therefore asymmetrically induced. On the other hand the $F_{1}$ and $\mathrm{F}_{2}$ in summer and winter $\left(\mathrm{F}_{2}\right.$ in summer from observations) are approximately intermediate in height between $\mathrm{L}$ and $\mathrm{S}$, that is, the plant height of $\mathrm{Pl}$ is not reconstituted in either environment on crossing $\mathrm{L}$ and $\mathrm{S}$, and this may be a permanent loss. The almost negligible interaction of plant height with the environment compared with that of plant weight is also found under field conditions, but since the weight differences are much greater, these are preferentially used, even though in different years and conditions the weight of $\mathrm{L}$ varies from two to ten times that of $\mathrm{S}$.

It is likely that the interactions between the genotrophs and environments in plant weight are themselves modified by the parental environments of the $\mathrm{L}, \mathrm{S}$ and $\mathrm{Pl}$ plants measured, through maternal effects or transient induced changes, so that the data in table 9 could be only part of a broader spectrum of interactions. It is appropriate here to qualify "stable" as applied to the large (L) and small (S) genotrophs. The word is used because these genotrophs remain consistently different from each other and from $\mathrm{Pl}$, showing no propensity for changes in inheritance as are shown by $\mathrm{Pl}$ when grown in different environments. There is evidence that small or moderate changes can be induced in them, which are thought to be transient, or semi-permanent, but to separate them from maternal effects requires more experimentation. The transmission of environmental effects through crosses, described in the previous section, is evidence that in some cases at least they are not maternal.

\section{INDUCED GHANGES IN THE VARIETY LYRAL PRINCE}

\section{(i) Induction}

The induced changes so far described were obtained in the variety Stormont Cirrus. Heritable changes have also been induced in another variety of flax, Lyral Prince. The same combinations of fertilisers were applied in the same year (1954) as in the initial induction experiments with Stormont Crirus. Due to shortage of space and equipment the plants were grown in smaller boxes and they were in another greenhouse so that the general inducing environment was different to that used for Stormont Cirrus. The first opportunity to grow the $C_{1}$ generation was in 1958, when the seeds were four years old, and the $C_{2}$ generation followed in 1959. Both $C_{1}$ and $C_{2}$ generations were transplanted into field plots receiving a compound fertiliser. The plant weights are given in table 10 .

There are significant differences $\left(P<I\right.$ per cent.) between the $C_{1}$ plant 
weights, and between the $C_{2}$. The $C_{1} / C_{2}$ correlation coefficient is 0.84 ( $\mathrm{P}<1$ per cent.). The $C_{2}$ values are more reliable and these show that apart from $n p k$, the pattern is the same as in Stormont Cirrus treated in the same year (compare tables 1 and 10). The highest value is given by $n$ and the lowest, but for $n p k$, by $n k ; p, k$ and $(l)$ are intermediate and $p k$ and $n p$ are somewhat larger, as in Stormont Cirrus. The exceptionally low value for $n p k$ is presumably due to the different general inducing environments.

TABle 10

Induction experiment with Lyral Prince. Mean plant weights (g.) in the $\mathrm{C}_{1}$ and $\mathrm{C}_{2}$ generations

$\begin{array}{rrrrrrrrr} & n p k & n p & n k & n & p k & p & k & (i) \\ C_{1} & 79 & 127 & 71 & 138 & 99 & 71 & 89 & 98 \\ C_{2} & 25 & 63 & 30 & 71 & 63 & 46 & 45 & 57\end{array}$

It was not practicable at the time to explore the stability of the eight types, but two extremes were selected, $n$ and $n k$, on the supposition that these would be respectively equivalent to large (L) and small (S) stable genotrophs of Stormont Cirrus although, because of the varietal difference, their genetic background may be different from that of $\mathrm{L}$ and $\mathrm{S}$ of Stormont Cirrus. They have in fact remained stable and are strikingly similar in appearance to $\mathrm{L}$ and $\mathrm{S}$ of Stormont Cirrus. This was borne out by nuclear DNA studies (Evans, 1968). As in Stormont Cirrus, L of Lyral Prince had more DNA, and $\mathrm{S}$ had less, than the original plastic variety Lyral Prince, although the overall difference between $\mathrm{L}$ and $\mathrm{S}$ was somewhat less, 12 per cent. compared with 16 per cent. between $\mathrm{L}$ and $\mathrm{S}$ of Stormont Cirrus.

TABLE $11 a$

Mean plant weights $(g$.$) of F_{1}$ families of a diallel cross between $L$ and $S$ genotrophs of Stormont Cirrus (S.C.) and Lyral Prince (L.P.)

\& $\begin{array}{cccccc}\text { S.C. } & \text { S.C. } & \text { L.P. } & \text { L.P. } \\ \text { S.C. } & \text { L } & 48 & 37 & 50 & 42 \\ \text { S.C. } & \text { S } & 43 & 18 & 36 & 18 \\ \text { L.P. } & \text { L } & 45 & 41 & 42 & 38 \\ \text { L.P. } & \text { S } & 39 & 21 & 39 & 20\end{array}$

(ii) A diallel cross between $L$ and $S$ genotrophs of Stormont Cirrus and Lyral Prince Although the L and S stable genotrophs of Lyral Prince (L.P.) are similar in many respects to $\mathrm{L}$ and $\mathrm{S}$ of Stormont Cirrus (S.C.), similar genetic changes may not have occurred in their induction, nor need they be at the same locus or loci. A diallel cross was made between them in 1959 to establish whether there was complementation, or any other form of interaction, between them.

The mean plant weights in the $F_{1}$ of the 16 crosses grown in 1960 are given in table $11 a$. The 16 crosses were set out in the field in a randomised block design with five replicates, five plants per plot, summing to 25 plants per cross. If the genetic difference between $\mathrm{L}$ and $\mathrm{S}$ is the same in the two varieties, S.C. and L.P., and if this is large compared with the difference 
between the genetic backgrounds of the two varieties, then provided there is no interaction between the two sets, $L$ and $S$ of one variety and $L$ and $S$ of the other, practically all the genetic variation within the diallel table would be the same as that given by a single gene difference. In which case if

TABLE $11 b$

Analysis of variance of reciprocal means

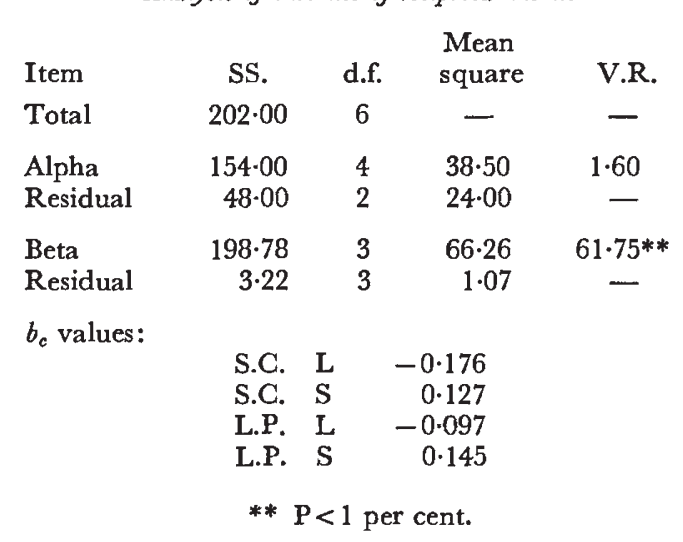

separate alpha and beta analyses (Durrant, 1969) of the data were made a good fit should be obtained with beta but not with alpha. The analyses in table $11 b$ show that applying the most critical test, employing the residual variation as error, beta is highly significant and accounts for practically the whole of the variation, whereas alpha, even without applying the correction

TABLE 12

Lyral Prince variety. Mean plant weights ( $g$.) and heights $(\mathrm{cm}$.$) of L, S, P l$ and $F_{1}$ of reciprocal crosses between $L$ and $S$ grown in summer and winter conditions. Nuclear $D N A$ values of $L, S$ and $P l$ in arbitrary units. Data taken from different experiments

\begin{tabular}{|c|c|c|c|c|c|}
\hline & $\mathbf{L}$ & $\mathrm{S}$ & P1 & $\mathrm{L} \times \mathrm{S}$ & $S \times L$ \\
\hline DNA & $95 \cdot 3$ & $86 \cdot 3$ & $90 \cdot 45$ & - & - \\
\hline \multicolumn{6}{|c|}{ Plant weight: } \\
\hline Summer & $\begin{array}{l}73 \cdot 5 \\
79 \cdot 6\end{array}$ & $\begin{array}{l}23 \cdot 6 \\
13 \cdot 4\end{array}$ & $16 \cdot 5$ & $\overline{42 \cdot 5}$ & $\overline{45 \cdot 4}$ \\
\hline Winter & $\begin{array}{l}14 \cdot 6 \\
17 \cdot 4\end{array}$ & $\begin{array}{l}10 \cdot 8 \\
12 \cdot 2\end{array}$ & $\begin{array}{l}8 \cdot 8 \\
-\end{array}$ & $\overline{18 \cdot 8}$ & $\overline{15 \cdot 4}$ \\
\hline \multicolumn{6}{|c|}{ Plant height: } \\
\hline Winter & $\begin{array}{l}130 \cdot 2 \\
133.7\end{array}$ & $\begin{array}{l}128 \cdot 2 \\
124 \cdot 0\end{array}$ & $\begin{array}{c}124 \cdot 0 \\
-\end{array}$ & $\overline{134 \cdot 7}$ & $\overline{131 \cdot 1}$ \\
\hline
\end{tabular}

required for parental error variation, is not. The $b_{c}$ values in table 11 show that both $\mathrm{L}$ genotrophs are dominant and both $\mathrm{S}$ genotrophs are recessive. Under the conditions of this experiment the difference between the mean parental plant weights of $\mathrm{L}$ and $\mathrm{S}$ of S.C. is somewhat larger than that of L.P. Nuclear DNA had a similar pattern. Therefore, as far as can be judged from this data there is no evidence of interaction, similar genetic changes 
have occurred in both varieties and the genetic background does not have a strong modifying effect.

\section{(iii) Genotroph/environment interaction}

Although the nuclear DNA changes are symmetrically induced, table 12 shows that the plant weights of $\mathrm{L}$ and $\mathrm{S}$ of this variety are not. In the summer growing-period $\mathrm{Pl}$ is well below $\mathrm{S}$ in plant weight and there is no interaction with the winter conditions such as to change their relative positions as occurred with Stormont Cirrus. The $F_{1}$ of the reciprocal crosses between $\mathrm{L}$ and $\mathrm{S}$ are intermediate in weight in the summer and, as far as can be judged, in the winter as well, so the Pl type is not reconstituted in the $\mathrm{F}_{1}$ in plant weight. Plant height was not recorded in the summer; in the winter it followed the same pattern as plant weight. On the assumption that the inducing environments were similar the difference in the symmetry of induction in Stormont Cirrus and Lyral Prince must be due to the genetic background which either modifies the inducing process or interacts, like the environment, with the induced change, unless Pl of Lyral Prince has changed during seed multiplication unaccompanied by change in nuclear DNA.

\section{Discussion}

Different environments are required for different purposes. Inducing environments induce heritable changes in plant weight in the plastic genotroph. Those inducing environments which produce the extreme forms, the large (L) and small (S) genotrophs in which no further large changes in plant weight have so far been induced, are also stabilising environments. These are compounded of a general inducing environment for stimulating optimal growth, and specific inducing environments for inducing the specific $\mathrm{L}$ and $\mathrm{S}$ genotrophs. Non-inducing environments ensure that the plastic genotroph does not change, nor become stabilised (i.e. does not lose its capacity to undergo induced change) when grown for seed multiplication or crossing purposes. They are therefore also non-stabilising environments. Standard environments are environments in which plants are normally grown and maximise the phenotypic differences between the genotrophs for observational and analytical purposes. They may or may not be inducing or stabilising. Further environments will have to be specified, for distinguishing between environments required in the early weeks of induction and those in later weeks, for those which induce changes in one character but not in another, and for those which induce only temporary, or transient, changes revealed by outcrossing or other techniques. The Earhart/Aberystwyth experiment shows that more care is required in organising the general component than the specific component of the inducing environment. The change in technique to growing $\mathrm{Pl}$ plants in 5-inch pots entirely in the greenhouse, and the gradual decline in fertility of the soil made up into compost, have been responsible for the smaller induced differences obtained in later years. Changes in the Pl genotroph during seed multiplication are being studied but they are believed to play a minor contributory role.

The characteristics of the different types of environments must be taken into account when planning the induction of heritable changes in other 
species of higher plants. The best material (Durrant, 1962) is probably inbred lines of a species from a natural population where during inbreeding, or in their multiplication, the lines are kept in environments considered to be non-inducing. The hypothesis is that a new genotype, produced by crossing, segregation, inbreeding or selection, may be immediately stable, or initially unstable becoming stable, or plastic where the plant retains the capacity for being pushed in one direction or another by some environments (inducing, perhaps stabilising), and continues to maintain its plasticity in others (noninducing). If the average, natural environment of a species is the best non-inducing environment then the species is maintaining an additional reserve of genetic variation which would be particularly advantageous to an inbreeder.

Varieties of crop plants are more likely to have become stabilised because they have been selected under agricultural conditions for superlatives in grain or foliage yield, which flax has not, and developmental stability may also be associated with genotrophic stability. The three inbred lines of Nicotiana rustica tested by Hill (1967), one of which showed induced changes, had been inbred for genetic studies rather than selected for commercial purposes. The hypothesis does not, however, draw a line between crop plants and plants from natural populations because some environments may induce smaller, more transient and less easily detected changes in selected varieties as well. The large induced changes are at one extreme, the developmental changes in the life of a plant may be at the other. In between are the less permanent changes induced in the garden pea (Highkin, 1958) by constant temperature.

A feature of the environmental induction is the dichotomy of the induced changes; either one stable type is induced or the other, depending on the environment. Judging by the induced changes in nuclear DNA, which do not appear to show any subsequent interaction with the environment, the dichotomy is symmetrical about the Pl type. The symmetry of plant weight and of other induced characters is either dependent upon the subsequent environment in which $\mathrm{L}, \mathrm{S}$ and $\mathrm{Pl}$ are grown, or is never symmetrical. The two dichotomous stable types, $\mathbf{L}$ and $\mathbf{S}$, only break down and show instability when they are brought together in the $\mathrm{F}_{1}$. At the same time the $H$ - $h$ locus heterozygous in the $F_{1}$, is also unstable and exhibits several levels of activity in the $F_{2}$ giving deviations from mendelian ratios in the $F_{3}$. It is conjecture what this dichotomy involves genically, and whether similar types of events occur in the differentiation of multicellular organisms, or would occur if hybrid tissues were obtained.

\section{SUMmary}

1. Differences among the progeny of flax plants of the variety Stormont Cirrus grown in different environments are environmentally induced heritable changes but only some environments are capable of inducing the two extreme forms of the $\mathrm{L}$ and $\mathrm{S}$ genotrophs.

2. The inducing environment is separated into a specific component for the induction of specific types of change (e.g. L or S), and a general component for promoting the optimal rate of growth necessary for the specific component to be effective. 
3. The maintenance of the plastic stock (Pl), in which the changes are induced, requires a multiplication environment which ensures that unwanted induced changes do not occur.

4. The $\mathrm{L}$ and $\mathrm{S}$ induced types are stable in themselves and no further major changes in plant weight can be induced in them. When, however, they are grown in inducing environments and at the same time crossed with PI grown in a non-inducing environment they are capable of transmitting changes induced by the environments to the $F_{1}$ of the crosses. Completed induced changes therefore involve at least two events.

5. Changes have been induced in a second variety, Lyral Prince. The $\mathrm{L}$ and $\mathrm{S}$ genotrophs of both varieties in a diallel cross showed no complementation and are similar in many, but not all, respects.

6. There is no evidence that variation between plants within the $L$ and $\mathrm{S}$ genotrophs is genetically determined.

7. Since Pl of Stormont Cirrus is intermediate in weight, the plant weights of $\mathrm{L}$ and $\mathrm{S}$ are symmetrically induced about P1. The $\mathrm{F}_{1}$ of reciprocal crosses between $\mathrm{L}$ and $\mathrm{S}$ is also intermediate so that the $\mathrm{Pl}$ plant weight is reconstituted on crossing. The genotrophs interact strongly with winter greenhouse conditions and heterotic effects are given not only by the heterozygous $\mathrm{F}_{1}$, but also by the homozygous $\mathrm{Pl}$, relative to $\mathrm{L}$ and $\mathrm{S}$. Plant height differences are not symmetrically induced nor are reconstituted in the $\mathrm{F}_{1}$.

Acknowledgments.-I am grateful to Professor Anton Lang for facilities provided at the Earhart Laboratories, Biology Division, California Institute of Technology, Pasadena. The work at the Earhart Laboratories was supported by a grant (G-17483) of the National Science Foundation. I am also grateful to Mrs Margaret K. Genthe for her technical assistance.

\section{References}

DURrant, A. 1962a. The environmental induction of heritable change in Linum. Heredity, $17,27-61$.

DURRANT, A. 1962b. Induction, reversion and epitrophism of flax genotrophs. Nature, 196, 1302-1304.

DURrant, A. 1969. Phenotype analysis of diallel crosses. Heredity, 24, 541-560.

DURRANT, A., AND Nicholas, D. B. 1970. An unstable gene in flax. Heredity, 25, 513-527. EVANs, G. M. 1968. Nuclear changes in flax. Heredity, 23, 25-38.

EVANS, G. M., DURRANT, A., AND REES, H. 1966. Associated nuclear changes in the induction of flax genotrophs. Nature, 212, 697-699.

HIGHKIN, H. R. 1958. Temperature induced variability in peas. Am. F. Botany, 45, 626-631. HILL, J. 1967. The environmental induction of heritable changes in Nicotiana rustica parental and selection lines. Genetics, 55, 735-754. 